\title{
Childbirth-Associated Fistula and Perineal Tears Repaired on Outreach Campaigns in Remote Democratic Republic of Congo
}

\author{
Justin Paluku ${ }^{1,2}$ \\ Phoebe Bruce ${ }^{3}$ \\ Eugénie Kamabu ${ }^{4}$ \\ Benjamin Kataliko' \\ Jonathan Kasereka ${ }^{5}$ \\ Annie Dube ${ }^{3}{ }^{3}$ \\ 'Department of Obstetrics and \\ Gynecology, HEAL Africa Tertiary \\ Hospital, Goma, North Kivu, Democratic \\ Republic of Congo; ${ }^{2}$ Department of \\ Obstetrics and Gynecology, University of \\ Goma (UNIGOM), Goma, North Kivu, \\ Democratic Republic of Congo; \\ ${ }^{3}$ Department of Undergraduate Medical \\ Education, Northern Ontario School of \\ Medicine, Sudbury, Ontario, Canada; \\ ${ }^{4}$ Department of Internal Medicine, HEAL \\ Africa Tertiary Hospital, Goma, North \\ Kivu, Democratic Republic of Congo; \\ ${ }^{5}$ Department of Surgery, HEAL Africa \\ Tertiary Hospital, Goma, North Kivu, \\ Democratic Republic of Congo
}

Purpose: To describe the demographics and evaluate the repair of childbirth-associated injuries leading to incontinence in patients in remote Democratic Republic of Congo (DRC). Patients and Methods: Four surgical outreach campaigns were organised between March 2018 and October 2019. These campaigns specifically targeted women with incontinence secondary to childbirth-associated injuries. Patients were recruited on a voluntary basis, and locations included Katako-kombe (Sankuru province), Wamba (Haut Uelé province), Karawa (North Ubangi province) and Kipaka (Maniema province). Necessary care was provided along with informal teaching with local healthcare professionals.

Results: A total of 481 patients, $14-71$ years old with a mean of 32 years of age, were included in this study. The average duration of incontinence associated with a childbirth injury was 6.8 years. Vesicovaginal fistulas $(277 / 481 ; 57.6 \%)$ and perineal tears $(148 / 481$; $30.8 \%$ ) were predominant, while the combination of vesicovaginal and rectovaginal fistulas were seen in only $5(1.0 \%)$ patients. Vaginal delivery $(259 / 481 ; 53.9 \%)$, caesarean section $(120 / 481 ; 25.0 \%)$ and laparotomy (hysterectomy) $(102 / 481 ; 21.2 \%)$ were identified as the causes of the fistulas treated during the surgical campaign. Vesicovaginal fistulas were more likely to require complex repairs, as compared to rectovaginal fistulas and perineal tears ( $P$-value $<0.0001)$. As well, vesicovaginal fistulas were less likely to result in successful resolution of the incontinence when compared to rectovaginal fistulas and perineal tears $(P$-value $<0.0001)$

Conclusion: Vesicovaginal fistulas are predominant among childbirth injuries encountered in remote DRC with poorly managed vaginal deliveries being the leading cause. Cure rate of fistulas and perineal tears is high during outreach campaigns; however, long-term follow-up is limited. There remains a need to train and mentor rural medical professionals in the DRC on adequate management of obstetric emergencies.

Keywords: vesicovaginal fistula, rectovaginal fistula, perineal tears, childbirth injuries

\section{Introduction}

Childbirth is relatively safe in the developed world; however, in developing countries many disparities in health outcomes and maternal complications exist. According to the WHO (2017) the lifetime risk of maternal death is as high as 1 in 45 in low-income countries, compared to 1 in 5400 in high income countries. In 2017, 295,000 women died following childbirth; Sub-Saharan Africa alone accounting for 196,000 of these deaths. ${ }^{1}$ Although there have been reductions in overall maternal mortality since 2000 , there remain significant challenges.
Correspondence: Justin Paluku Department of Obstetrics and Gynecology, HEAL Africa Tertiary Hospital, P.O. Box 319, Goma, Democratic Republic of Congo Email justin.paluku@healafrica.org 
Beyond maternal mortality, many women in lower resource countries experience postpartum complications including genital fistulas and perineal tears. These morbidities can be debilitating for the woman who experience them, especially when combined with limited access to the medical facilities and expertise required for repair. ${ }^{2}$

One of the more devastating outcomes of genital fistula and perineal tears is either urinary and/or fecal incontinence. Multiple studies have shown that in addition to the physical consequences of incontinence, women will often experience social and religious exclusion, stigma, and divorce as a result of this condition. ${ }^{3-6}$

Obstetric genital fistula, specifically the vesicovaginal fistula (VVF), is a consequence of prolonged and traumatic labour. ${ }^{7}$ This type of labour leads to necrosis and sloughing of the maternal vaginal septum secondary to prolonged pressure from the fetal head. ${ }^{7}$ While not all genital fistulas are caused by this mechanism, obstructed labour is by far the most common cause in low resource countries. $^{2,3,7-9}$ A less prevalent cause of childbirthassociated fistulas is surgical error (eg perforation of bladder during caesarean section or hysterectomy). ${ }^{10}$ Key factors to ameliorate this complication include: reducing delays in care access, providing skilled and appropriate care, and improving preventative and public health strategies. ${ }^{11}$

In the Democratic Republic of Congo (DRC), decades of civil conflict, extreme poverty, and poor health infrastructure have all negatively impacted maternal care. In particular, the DRC is known to have a maternal mortality ratio of 550 to 100,000 live births and as of 2018, more than 42,000 women are awaiting surgical care for genital fistulas alone. ${ }^{12}$ This problem is compounded by the fact that the majority of DRC's population resides in rural areas, where qualified medical care is even more scarce. ${ }^{13}$ Despite only accounting for approximately $30 \%$ of the DRC population, the 2014 Demographic and Health Survey estimated that $80 \%$ of all skilled birth attendance and well-equipped facilities are located in urban areas. ${ }^{13}$

Established in 2000, HEAL Africa Tertiary Hospital is a non-governmental organization providing medical care and social support to the residents of Goma, in North-Kivu province as well as more broadly through outreach campaigns to other provinces in remote DRC. ${ }^{14}$ The hospital offers a full range of services, including general surgery, orthopaedics, obstetrics and gynaecology, paediatrics, internal medicine, radiology and pathology services, in addition to research and training opportunities for healthcare professionals. ${ }^{14}$ HEAL Africa also coordinates community-based initiatives supporting development, female empowerment, and public health promotion. Approximately 10 outreach campaigns are organized through HEAL Africa Tertiary Hospital annually. ${ }^{14}$ The purpose of these campaigns varies based on community need; however, largely focus on healthcare and specialty services, such as fistula repair, for women who would otherwise remain uncared for.

The purpose of this study is to document childbirthassociated fistula and perineal tears seen in rural communities during outreach campaigns and to evaluate features of these injuries as well as their surgical repair outcomes.

\section{Methods}

\section{Setting}

Medical team for outreach campaigns originated from the HEAL Africa Tertiary Hospital located in Goma, the capital city of North-Kivu province, and travelled to four remote communities in surrounding provinces. Locations were selected by request of local rural health authorities, based on previously identified gaps in care for women in their own communities. Selected locations had high numbers of potential patients and lacked appropriate, specialized, surgical services related to fistula repair. As well, local engagement and support of the campaign were crucial in location selection and success of the project.

\section{Study Design and Data Collection}

To better understand childbirth related fistula and the associated challenges and barriers to care faced by women in rural DRC communities, a team of healthcare providers from HEAL Africa Tertiary Hospital collected descriptive data during four surgical outreach campaigns. Outreach locations included Karawa (North Ubangi province), Katako-Kombe (Sankuru province), Wamba (Haut Uele province) and Kipaka (Maniema province) and care was provided at local hospitals in each region. The healthcare team from HEAL Africa consisted of two gynecologists, one operating room nurse and one anesthetic nurse. The HEAL Africa team also relied on, and worked closely with, local doctors, nurses, and staff in each location. Visiting team members had more than 10 years of experience attending to fistula patients in both urban and remote settings. Outreach campaigns ran from March 2018 until October 2019 and spent approximately three to four weeks at each location. 
Prior to the start of the campaign, communities were informed via local radio broadcasts and churches of the opportunity to receive medical care for childbirthassociated fistulas and perineal tears. Women experiencing fecal and/or urinary incontinence following delivery, surgical procedure or traumatic event related to childbirth were encouraged to attend the outreach campaign. Inclusion criteria included a history of urinary and/or fecal incontinence after vaginal or caesarean section (C-section) delivery, hysterectomy or laparotomy related to childbirth complications. Additionally, surgical fitness, which involved evaluation of overall health status, existing comorbidities, and hemodynamic stability, was a requirement for participants. Women with urinary and/ or fecal incontinence unrelated to childbirth were provided with necessary care but were excluded from this study. Patients suspected of malignancy were also excluded from the study but did receive appropriate care and coordination of future services.

Informed consent was obtained from each woman who met the inclusion criteria and desired to be involved in the study. At each location, HEAL Africa's medical team worked with local healthcare teams to provide necessary care to local women. Data collection included age and duration of incontinence, type of incontinence and the cause of incontinence (vaginal delivery, C-section, or laparotomy). Identified injuries were classified as VVF, perineal tears, rectovaginal fistula (RVF) or VVF and RVF. The type of repair (VVF, perineal tear, RVF or VVF and RVF) was noted and the complexity of surgery was assessed.

Surgical complexity was assessed by extent of scar tissue present and the ability to remobilize remaining healthy tissue. A repair was deemed simple if the defect was easily accessible with little to no scar tissue, moderate if accessible with moderate scar tissue and complex if difficult to access due to scar tissues and/or little to no healthy tissue to mobilize from extensive damage. Surgical time also represented a factor in determining surgical complexity. A simple repair had operating times $<30$ minutes, moderate $<$ two hours, and complex $>$ two hours.

Post-intervention, a urinary catheter was placed in patients with VVF and they were instructed to leave it in place for 14 days. Those repaired for RVF and perineal tears were instructed to keep the urinary catheter in for three days. Throughout the hospital stay, patients were monitored daily. Those with VVF were instructed to drink four to five liters of water each day to ensure clear urine drains from their bladders. Hospital nurses monitored patient beds and sheets to ascertain dryness. Additionally, monitoring for continence, the ability to urinate, and surgical wound assessment was conducted daily. Post-operative complications, which included wound infection, hemorrhage, catheter blockade, and/or spinal headache, were noted along with the duration of hospital stay.

All data were anonymized, recorded in French on paper forms, and upon returning to HEAL Africa Tertiary Hospital, entered into a Microsoft Excel spreadsheet by an outreach team member.

\section{Analysis}

In May 2020, after outreach campaigns were complete, anonymized data were translated to English, grouped according to injury type (VVF, RVF, VVF and RVF or perineal tear) and basic descriptive and statistical analyses were performed. Descriptive analysis was completed using Microsoft Excel and statistical analysis was done using SAS Studio (Release: 3.8 Basic Edition, Site name: University Edition $2.89 .4 \mathrm{M} 6$ ). Both Chi square tests and Fisher's Exact tests (when cell count was less than five) were used for stated p-values.

\section{Ethical Considerations}

Informed consent was received from eligible women. All data was anonymized, and identifiers were excluded to ensure confidentiality of the participants. The study was designed and conducted by researchers from HEAL Africa's urogynecology team and approved by HEAL Africa Tertiary Hospital Ethics Committee. This study was conducted in accordance with the Declaration of Helsinki.

\section{Results}

Between March 2018 and October 2019, four outreach campaigns provided care to a total of 481 female patients with history of urinary, fecal or combined incontinence related to childbirth. Patient age ranged from 14 to 71 years with a mean age of 32 years. The mean duration of incontinence was 6.8 years with a range 0.1 to 43 years.

\section{Injury Characteristics}

Urinary incontinence was reported by 269 (55.9\%) patients while fecal incontinence was reported by 197 (41\%) patients. A smaller number of patients, 15 or $3.1 \%$, reported both urinary and fecal incontinence. 


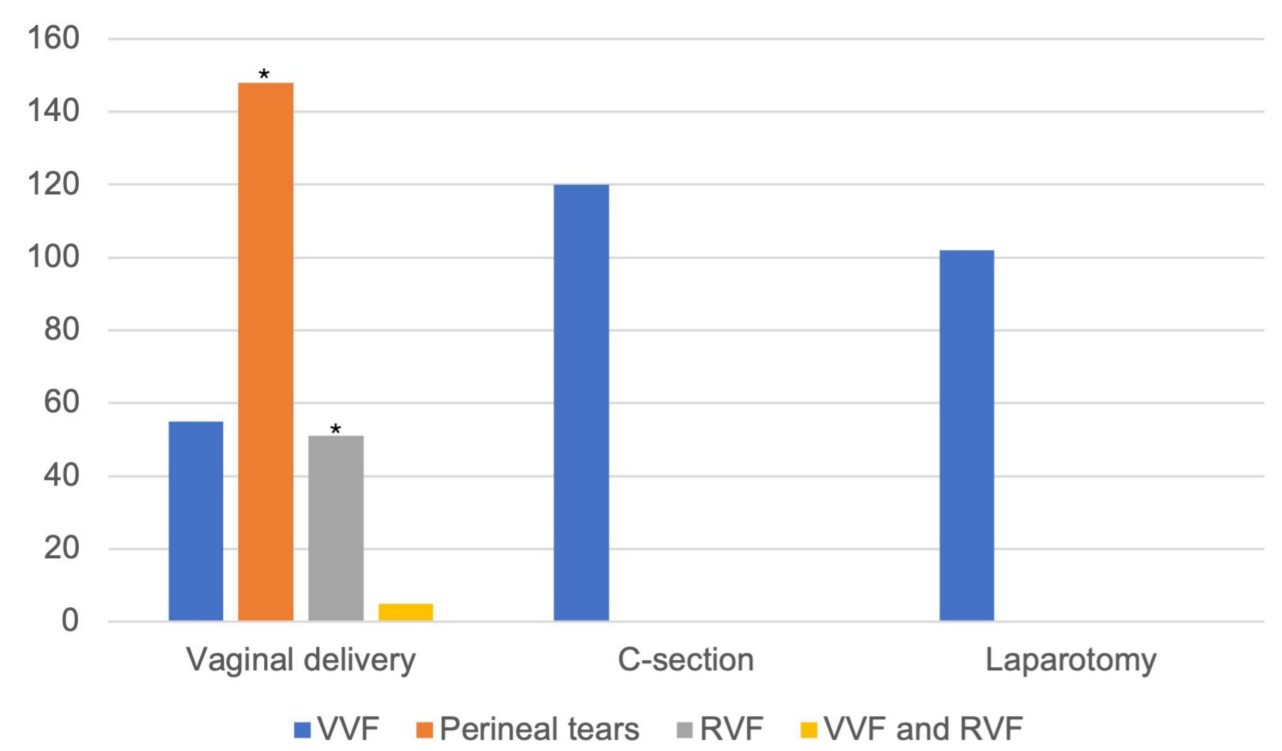

Figure I Type and cause of childbirth-associated incontinence.

Note: $* P$-value $<0.001$.

The majority of patients seen on outreach presented with VVF (277 patients, 57.6\%) or perineal tears (148 patients, $30.8 \%)$. A smaller number of patients presented with RVF (51 patients, $10.6 \%)$ or a combination of both VVF and RVF (5 patients, $1.0 \%$ ). Vaginal delivery was the leading cause of childbirth-associated incontinence $(\mathrm{n}=259,53.9 \%)$, while surgical procedures relating to childbirth were identified as the cause in 222 patients (C-section: $n=120$, $25.0 \%$, laparotomy: $n=102,21.2 \%$ ).

As seen in Figure 1, C-sections and laparotomies were only associated with VVFs, and perineal tears and RVFs were more likely to result from vaginal deliveries (p-value $<$ 0.0001).

\section{Surgical Repairs, Outcomes and Hospital Stays}

Of the total 481 campaign participants, $276(57.4 \%)$ VVF repairs, $148(30.8 \%)$ perineal tear repairs, 51 (10.6\%) RVF repairs and $3(0.6 \%)$ dual VVF and RVF repairs were performed. Only $3(0.6 \%)$ patients' injuries were deemed inoperable.

Of the 478 operable cases, $10(2.1 \%)$ patients underwent the procedure using general anaesthesia, while 468 (97.9\%) patients received local anaesthetic at the repair site. Surgical difficulty was deemed simple in 261 cases (54.6\%), moderate in 147 cases (30.8\%) and complex in 70 cases $(14.6 \%)$.
After surgical repair, 422 females $(87.7 \%)$ were declared dry and no longer suffered from incontinence. Unfortunately, for 56 patients (11.7\%) surgical repairs were unsuccessful and incontinence was still experienced at the time of discharge from the hospital.

VVFs were more likely to require complex repairs, as compared to RVFs and perineal tears (p-value $<0.0001$ ). As well, VVFs were less likely to result in successful resolution of the incontinence when compared to RVFs and perineal tears ( $p$-value $<0.0001)$. Further details can be seen in Table 1.

Finally, duration of hospital stay ranged from 1 to 21 days with a mean duration of 11.2 days. A total of 391 patients $(81.8 \%)$ did not report any complication after surgery, whereas, wound infections were seen in 36 patients (7.5\%) and 25 patients (5.2\%) haemorrhaged, however, did not require a blood transfusion.

\section{Discussion}

This study describes the injuries that women in remote DRC develop secondary to childbirth, using the inclusion criteria of incontinence to recruit patients for surgical intervention. For the patients in this study, VVF was the most common complication, and urinary incontinence was the most common complaint. Unfortunately, VVF was also more likely to require a complex repair, with a correspondingly lower resolution rate.

While the majority of injuries seen in this study were secondary to vaginal delivery, a not insignificant 
Table I Characteristics of Surgical Repairs and Outcomes

\begin{tabular}{|c|c|c|c|c|c|c|}
\hline & \multicolumn{4}{|c|}{ Type of Repair } & \multirow[t]{2}{*}{ P-value } & \multirow{2}{*}{$\begin{array}{c}\text { Total } \\
\text { N (\% of } 478)\end{array}$} \\
\hline & $\begin{array}{c}\text { VVF } \\
\text { N (\% of } 276)\end{array}$ & $\begin{array}{l}\text { Perineal Tear } \\
\mathbf{N}(\% \text { of } 148)\end{array}$ & $\begin{array}{c}\text { RVF } \\
\mathbf{N}(\% \text { of } \mathbf{5 I})\end{array}$ & $\begin{array}{c}\text { VVF and RVF } \\
\mathbf{N} \text { (\% of 3) }\end{array}$ & & \\
\hline \multicolumn{7}{|l|}{ Difficulty } \\
\hline Simple & $107(38.6)$ & $122(82.4)$ & $32(62.7)$ & - & $<0.0001$ & $26 \mid(54.6)$ \\
\hline Moderate & $110(39.7)$ & $22(14.9)$ & $14(27.5)$ & I (33.3) & & 147 (30.8) \\
\hline Complex & $59(21.4)$ & $4(2.7)$ & $5(9.8)$ & $2(66.6)$ & & $70(14.6)$ \\
\hline \multicolumn{7}{|l|}{ Outcome } \\
\hline Successful: dry & $227(82.2)$ & $148(100.0)$ & $44(86.3)$ & $3(100.0)$ & $<0.0001$ & $422(88.3)$ \\
\hline Unsuccessful & $49(17.8)$ & - & $7(13.7)$ & - & & $56(11.7)$ \\
\hline Total Operable Cases & $276(100.0)$ & $148(100.0)$ & $5 I(100.0)$ & $3(100.0)$ & & $478(100.0)$ \\
\hline
\end{tabular}

Note: Although there were $48 \mathrm{I}$ participants, 3 cases were deemed inoperable, $N=478$.

proportion can be attributed to surgical error from C-section or laparotomy. In particular, VVF was the most common cause of incontinence after an operative delivery. This study corroborates the need to train more medical personnel to enable them to safely attend to emergency obstetric conditions as found in previous studies. ${ }^{8,9}$

The development of a VVF during vaginal delivery is multifactorial; however, it comes as no surprise that the frequency of this complication is exacerbated by poor preventative care. For example, observers during this study noted that often patients in labour do not benefit from regular bladder emptying. This leads them to reach full dilatation of the cervix with a full bladder. The full bladder is therefore highly exposed to injuries during the second stage of labour. Again, highlighting the need for trained birth attendants to reduce childbirth-associated injuries.

This study also found that patients who presented with complaint of urinary and/or fecal incontinence had been symptomatic for anywhere from 0.1 to 43 years (median of 6.8 years). Similar findings were recently reported in a study that compiled data on patients treated on outreach for genitourinary fistula during a 5 year timeframe (20132018). ${ }^{12}$ In a study conducted in northern DRC on obstetric fistulas, the mean duration of the illness before repair during surgical campaigns, was 8.4 years. ${ }^{15}$ Our study adds to the growing body of literature highlighting a longstanding need for fistula related care for women in rural and remote areas.

Although in this study the successful outcome proportion was quite high $(87.7 \%)$, surgical repair was more difficult in patients who had waited longer for their repair. Fibrosis is more likely to occur in older injuries and, surgeries were more likely to be complicated. Studies from other African countries like Uganda, Tanzania and Kenya have showed that closure rate is higher in cases that are repaired within 3 months from development of the fistula. ${ }^{16}$ For sustainability and better outcomes long term, it is essential to combine training with mentoring and ongoing supervision. It is also crucial for women with obstructed labor to be able to quickly access a health facility with qualified staff and appropriate infrastructure to provide high-quality emergency obstetric care. ${ }^{9}$

An important extension of this study involves examining the causes of childbirth-associated fistula and perineal tears to identify strategies for safe birthing practices. In 2012, Dr. Lewis Wall used a Haddon Matrix to examine factors influencing obstetric fistulas in low-resource countries. ${ }^{7}$ Short-term strategies included "enhanced surveillance of labor, improved access to emergency obstetrical services (particularly cesarean delivery), competent medical care for women both during and after obstructed labor, and the development of specialist fistula centers to treat injured women where fistula prevalence is high." ${ }^{77}$ Long-term strategies focused primarily on education and system reform, highlighting the necessary political involvement at both the individual country and international level. Strategies included "universal access to emergency obstetric care, improved access to family planning services, increased education for girls and women, community economic development, and enhanced gender equity."7

As a result of the high prevalence and significant morbidity seen in the study, Heal Africa surgical outreach teams can utilize these recommendations to optimize the 
positive impact these campaigns have on the remote communities they serve. Specifically, outreach teams could include additional healthcare practitioners who focus on capacity building and knowledge translation while serving the remote communities. Such providers may host workshops and seminars to local birth attendants on prenatal care, labour surveillance, care during and after obstructed labor, and early involvement of emergency services, as well as to local women and girls on focusing on empowerment, economic development, prenatal care, and family planning.

This study had several limitations. Although it gives a broad picture of childbirth related fistulas and perineal tears in remote DRC, the voluntary nature of the study and participant recruitment criteria has the potential to introduce bias. Also, the study does not have an adequately long follow-up period to assess patients for the long-term outcomes of surgical repairs. Ideally, to assess long-term impact on the community and the efficacy of local involvement, a follow up study should be considered.

In countries like the DRC, fistula care is usually offered during surgical campaigns organized by NGOs, such as HEAL Africa. While this is undoubtedly better than nothing, the short-term nature of these campaigns can lead to inconsistency in level of care, experience and follow up. As such, local participation is essential to longterm resolution of these issues. Further research and investigation into the feasibility of providing education and intervention in the early stages of labour to prevent complication also needs to be done.

\section{Conclusion}

Vesicovaginal fistulas are predominant among childbirth-associated injuries encountered in remote DRC. While their leading cause was vaginal delivery, iatrogenic errors caused nearly half of identified injuries. Cure rate of fistulas and perineal tears is high during outreach campaigns; however, outcomes were poorer the longer the patient waited for repair. There is a need to train, mentor and support medical professionals working in remote DRC. There is need to train, mentor and continuously supervise medical professionals working in remote DRC on adequate management of obstetric emergencies. A lack of skilled professionals appears to be one of the underlying causes of childbirth injuries.

\section{Acknowledgments}

The authors are grateful to the women in remote DRC who have been living with childbirth-associated fistulas, often for many years, and chose to seek care from our surgical outreach campaign. We are also indebted to the local healthcare teams across the various communities in remote DRC. This study would not have been possible without the support of HEAL Africa Tertiary Hospital. The surgical outreach campaigns from HEAL Africa Tertiary Hospital are funded by the Fistula Foundation.

\section{Author Contributions}

All authors made a significant contribution to the work reported, whether that is in the conception, study design, execution, acquisition of data, analysis and interpretation, or in all these areas; took part in drafting, revising or critically reviewing the article; gave final approval of the version to be published; have agreed on the journal to which the article has been submitted; and agree to be accountable for all aspects of the work.

\section{Disclosure}

Dr Benjamin Kataliko Snr reports a patent Safe motherhood licensed to Childbirth surgery, a patent Cesarean section licensed to Childbirth surgery, a patent Prevention and control of infections licensed to Childbirth surgery. The authors report no other conflicts of interest in this work.

\section{References}

1. World Health Organization. Maternal mortality; September 19, 2019. Available from: https://www.who.int/news-room/fact-sheets/detail/ maternal-mortality. Accessed May 13, 2021.

2. Wall LL. Obstetric vesicovaginal fistula as an international public-health problem. Lancet. 2006;368(9542):1201-1209. doi:10.1016/S0140-6736(06)69476-2

3. Arrowsmith S, Hamlin EC, Wall LL. Obstructed labor injury complex. Obstet Gynecol Surv. 1996;51(9):568-574. doi:10.1097/00006254199609000-00024

4. Muleta M, Hamlin EC, Fantahun M, Kennedy RC, Tafesse B. Health and social problems encountered by treated and untreated obstetric fistula patients in rural Ethiopia. J Obstet Gynaecol Canada. 2008;30 (1):44-50. doi:10.1016/S1701-2163(16)32712-8

5. Browning A, Fentahun W, Goh JTW. The impact of surgical treatment on the mental health of women with obstetric fistula. BJOG: Int J Obstet Gynaecol. 2007;114(11):1439-1441. doi:10.1111/j.14710528.2007.01419.x

6. Adler AJ, Ronsmans C, Calvert C, Filippi V. Estimating the prevalence of obstetric fistula: a systematic review and meta-analysis. BMC Pregnancy Childbirth. 2013;13:246. doi:10.1186/1471-2393-13-246

7. Wall LL. Preventing obstetric fistulas in low-resource countries. Obstet Gynecol Surv. 2012;67(2):111-121. doi:10.1097/OGX.0b013e3 182438788 
8. Egziabher TG, Eugene N, Ben K, Fredrick K. Obstetric fistula management and predictors of successful closure among women attending a public tertiary hospital in Rwanda: a retrospective review of records. BMC Res Notes. 2015;8:774. doi:10.1186/s13104-015-1771-y

9. Raassen TJIP, Ngongo CJ, Mahendeka MM. Iatrogenic genitourinary fistula: an 18-year retrospective review of 805 injuries. Int Urogynecol J. 2014;25(12):1699-1706. doi:10.1007/s00192-0142445-3

10. Smith W. Vesicovaginal fistula. BJU Int. 1999;83(5):564-570. doi:10.1046/j.1464-410x.1999.00006.x

11. Maheu-Giroux M, Filippi V, Maulet N, et al. Risk factors for vaginal fistula symptoms in Sub-Saharan Africa: a pooled analysis of national household survey data. BMC Pregnancy Childbirth. 2016;16:82. doi:10.1186/s12884-016-0871-6

12. Maroyi R, Keyser L, Hosterman L, Notia A, Mukwege D. The mobile surgical outreach program for management of patients with genital fistula in the Democratic Republic of Congo. Int J Gynecol Obstet. 2020;148(S1):27-32. doi:10.1002/ijgo.13036
13. Ministère du Plan et Suivi de la Mise en œuvre de la Révolution de la Modernité (MPSMRM/Congo), Ministère de la Santé Publique (MSP/Cong), ICF International. Enquête Démographique et de Santé en République Démocratique du Congo 2013-2014 (EDSRDC II); 2014. Available from: http://dhsprogram.com/pubs/pdf/ FR300/FR300.pdf. Accessed October 12, 2021.

14. HEAL Africa. HEAL Africa - our story; 2017. Available from: https://healafrica.org/our-story/. Accessed May 19, 2020.

15. Paluku JL, Carter TE. Obstetric vesico-vaginal fistulae seen in the Northern Democratic Republic of Congo: a descriptive study. Afr Health Sci. 2015;15(4):1104-1111. doi:10.4314/ahs.v15i4.8

16. Raassen TJIP, Verdaasdonk EGG, Vierhout ME. Prospective results after first-time surgery for obstetric fistulas in East African women. Int Urogynecol J. 2008;19(1):73-79. doi:10.1007/s00192-007-0389-6

\section{Publish your work in this journal}

The International Journal of Women's Health is an international, peerreviewed open-access journal publishing original research, reports, editorials, reviews and commentaries on all aspects of women's healthcare including gynecology, obstetrics, and breast cancer. The manuscript management system is completely online and includes a very quick and fair peer-review system, which is all easy to use. Visit http://www.dovepress.com/testimonials.php to read real quotes from published authors. 